\title{
Wideband Time Reversal of Microwave Signals Based on Phase Conjugating
}

\author{
Cheng-Guang SUN ${ }^{1,2}$, Jia-Lin $L I^{1}$ \\ ${ }^{1}$ School of Physics, University of Electronic Science and Technology of China, \\ No. 4, Sec. 2, North Jianshe Road, Chengdu 610054, China \\ ${ }^{2}$ Institute of Antenna and Microwave Techniques, Tianjin University of Technology and Education, \\ No.1310, Dagu South Road, Tianjin 300222, China \\ sunchengguang23@gmail.com,jialinli@uestc.edu.cn \\ Submitted December 31, 2017 / Accepted July 16, 2018
}

\begin{abstract}
A phase conjugating network to realize wideband time reversal of microwave signals is studied in this paper. After discussions on the operation principle of the reversed signal in the time and frequency domains, a prototype network based on phase-conjugating mixing is developed. A demonstrator is designed and fabricated, and two examinations are carried out to confirm the presented method. For a fixed LO signal, a chirp RF signal with $40-\mathrm{MHz}$ bandwidth can be phase-conjugated mixing, thus time reversed effectively. To widen the operation bandwidth, a method named dynamically synchronous phase conjugating is proposed; this enables a microwave signal to be dynamically reversed by synchronously varying the $L O$ frequency along with the $R F$ frequency, thus achieving the phase-conjugating dynamically in a wide frequency band. The simulation and experiment results of a wideband microwave signal, ranging from 5.4 to $6.25 \mathrm{GHz}$ under a measured conversion loss of $\leq 10 \mathrm{~dB}$, verify our studies.
\end{abstract}

\section{Keywords}

Time reversal, phase conjugating, mixing, synchronism

\section{Introduction}

It has been shown the time reversal can exhibit some unique characteristics involving the temporal and spatial focusing and super-resolution focusing [1-4], thus having many potential applications in detection, imaging, monitoring and wireless communications [5-9]. The key issue of a time reversal based system is how to effectively reverse a required signal in the time domain. For a monochromatic signal, it is known the time reversal corresponds to phase conjugating in the frequency domain [10], [11], and it was found in applications to the retrodirective array [12-14]. For a narrow band signal like a chirp pulse, the phase-conjugating method can also achieve the time reversal at mi- crowave frequencies since its operation is near the center frequency. But for some applications like the target detection, the detection signal generally occupies a certain frequency band in the spectrum [15]. In this case, time reversal of this kinds of signal becomes a challenge and is worthy of studying in-depth.

In this paper, we discuss a new method to time reverse a microwave signal with a wide frequency range. Theories of the reversed signal in the time and frequency domains are formulated firstly. To verify the analyses, a phase-conjugating network is developed and examined experimentally. Measurements on a chirp signal with a bandwidth of $40 \mathrm{MHz}$ are performed and good performance is observed. Further, harmonic signals sweeping from 5.4 to $6.25 \mathrm{GHz}$ with dynamically synchronized LO frequencies from 10.8 to $12.5 \mathrm{GHz}$ are investigated experimentally, and results validate this study.

\section{Operation Principles}

Time reversal of a monochromatic signal can be achieved based on the phase conjugating. For a monochromatic real signal $f(t)$, let its angular frequency to be $\omega_{0}$. The Fourier transformation has the following relations

$$
F\left(\omega_{0}\right)=\int_{-\infty}^{\infty} f(t) \mathrm{e}^{-\mathrm{j} \omega_{0} t} \mathrm{~d} t \Leftrightarrow f(t),
$$

and simply, we have

$$
F^{*}\left(\omega_{0}\right)=\int_{-\infty}^{\infty} f(t) \mathrm{e}^{j \omega_{0} t} \mathrm{~d} t=\int_{-\infty}^{\infty} f(-t) \mathrm{e}^{-j \omega_{0} t} \mathrm{~d} t \Leftrightarrow f(-t) .
$$

Compared with (1) and (2), one can see $f(t)$ and $f(-t)$ correspond to the phase conjugation in the frequency domain. Now, considering a real signal $f(t)$ has some bandwidth in the spectrum, and its angular frequency is ranged from $\omega_{0}-\Delta \omega$ to $\omega_{0}+\Delta \omega$, then its Fourier transformation takes the form

$$
F\left(\omega_{0} \pm \Delta \omega\right)=\int_{-\infty}^{\infty} f(t) \mathrm{e}^{-j\left(\omega_{0} \pm \Delta \omega\right) t} \mathrm{~d} t
$$




$$
\begin{aligned}
F^{*}\left(\omega_{0} \pm \Delta \omega\right) & =\int_{-\infty}^{\infty} f(t) \mathrm{e}^{j\left(\omega_{0} \pm \Delta \omega\right) t} \mathrm{~d} t \\
& =\int_{-\infty}^{\infty} f(-t) \mathrm{e}^{-j\left(\omega_{0} \pm \Delta \omega\right) t} \mathrm{~d} t \Leftrightarrow f(-t) .
\end{aligned}
$$

This means the phase-conjugating method can also be applicable to time reverse a signal with a certain bandwidth in its spectrum.

In engineering, realization of the phase conjugating is generally based on the frequency mixing, as shown in Fig. 1, where the central angular frequencies of radio frequency (RF) and local oscillator (LO) are $\omega_{0}$ and $2 \omega_{0}$, respectively. Now, we first consider the RF is a chirp signal, given by

$$
f_{\mathrm{RF}}(t)=A \cos \left(\omega_{0} t+K t^{2} / 2+\varphi\right)
$$

where $K$ is the slope of frequency tuning, and the LO signal is described by

$$
f_{\mathrm{LO}}(t)=B \cos \left(2 \omega_{0} t\right) .
$$

After mixing, the intermediate frequency (IF) signal would be

$$
f_{\mathrm{IF}}(t)=C \cos \left(\omega_{0} t-K t^{2} / 2-\varphi\right)+C \cos \left(3 \omega_{0} t+K t^{2} / 2+\varphi\right) .
$$

With a low-pass filtering, one can see

$$
f_{\mathrm{IF}}(t)=f_{\mathrm{RF}}(-t)=D \cos \left(\omega_{0} t-K t^{2} / 2-\varphi\right) .
$$

Thus time reversal of a chirp signal can be realized in this mixing process. If the RF signal is sinusoidal with a frequency band ranging from $\omega_{0}-\Delta \omega$ to $\omega_{0}+\Delta \omega$, given by

$$
f_{\mathrm{RF}}(t)=A \cos \left[\left(\omega_{0} \pm \Delta \omega\right) t+\varphi\right],
$$

while the LO signal is dynamically changed to

$$
f_{\mathrm{LO}}(t)=B \cos \left[2\left(\omega_{0} \pm \Delta \omega\right) t\right] .
$$

After mixing, the IF signal becomes

$$
f_{\mathrm{IF}}(t)=C \cos \left[\left(\omega_{0} \pm \Delta \omega\right) t-\varphi\right]+C \cos \left[3\left(\omega_{0} \pm \Delta \omega\right) t+\varphi\right]
$$

With the low-pass filtering, we have

$$
f_{\mathrm{IF}}(t)=f_{\mathrm{RF}}(-t)=D \cos \left[\left(\omega_{0} \pm \Delta \omega\right) t-\varphi\right] .
$$

This result indicates the mixing process enables time reversal of a microwave signal with a certain frequency bandwidth to be achievable.

The phase-conjugating network presented here consists of a ring hybrid, a branch-line balun and mixing com-

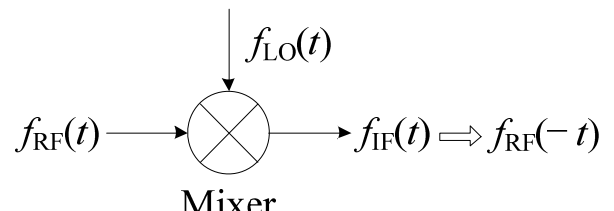

Fig. 1. Schematic diagram of time reversal a microwave signal based on frequency mixing. ponents, as shown in Fig. 2. The center frequencies of the ring hybrid and the branch-line balun are $f_{0}$ and $2 f_{0}$, respectively. The RF signal is applied to port 1 , and the LO signal is applied to port 5. For a chirp RF signal, the LO signal is set as described in (6). After mixing, one can find the mixing components to be

$\mathrm{MX}_{1}(t)=$

$C^{\prime}\left[\cos \left(\omega_{0} t-K t^{2} / 2-\varphi+\pi\right)+\cos \left(3 \omega_{0} t+K t^{2} / 2+\varphi-2 \pi\right)\right]$,

and

$\mathrm{MX}_{2}(t)=$

$C\left[\cos \left(\omega_{0} t-K t^{2} / 2-\varphi-\pi\right)+\cos \left(3 \omega_{0} t+K t^{2} / 2+\varphi-2 \pi\right)\right]$.

The IF signal at port 4 would be

$f_{\mathrm{IF}}(t)=$

$C\left[\cos \left(\omega_{0} t-K t^{2} / 2-\varphi+\pi / 2\right)+\cos \left(3 \omega_{0} t+K t^{2} / 2+\varphi-\pi / 2\right)\right] .(15)$

By a low-pass filtering, we have

$$
f_{\mathrm{IF}}(t)=D \cos \left(\omega_{0} t-K t^{2} / 2-\varphi+\pi / 2\right)
$$

Compared with the input signal in (5), the chirp signal is time reversed after this network. The additional phase of $f_{\mathrm{IF}}(t)$ is attributed to the network structure that does not effect on the reversed waveform. At port 4, the RF leakage from two mixing components is

$f_{\mathrm{RF}}(t)=$

$D^{\prime}\left[\cos \left(\omega_{0} t+\pi K t^{2}+\varphi-2 \pi\right)+\cos \left(\omega_{0} t+\pi K t^{2}+\varphi-\pi\right)\right]=0$.

If RF and LO signals are respectively chosen as the ones in (9) and (10), results for the mixing components would be:

$\operatorname{MX}_{1}(t)=$

$C^{\prime}\left\{\cos \left[\left(\omega_{0} \pm \Delta \omega\right) t-\varphi+\pi\right]+\cos \left[3\left(\omega_{0} \pm \Delta \omega\right) t+\varphi-2 \pi\right]\right\}$,

and

$\mathrm{MX}_{2}(t)=$

$C^{\prime}\left\{\cos \left[\left(\omega_{0} \pm \Delta \omega\right) t-\varphi-\pi\right]+\cos \left[3\left(\omega_{0} \pm \Delta \omega\right) t+\varphi-2 \pi\right]\right\}$.

When observed at port 4 , we have

$f_{\mathrm{IF}}(t)=$

$C\left\{\cos \left[\left(\omega_{0} \pm \Delta \omega\right) t-\varphi+\pi / 2\right]+\cos \left[3\left(\omega_{0} \pm \Delta \omega\right) t+\varphi-\pi / 2\right]\right\}$

Further, by a low-pass filtering, it is seen

$$
f_{\mathrm{IF}}(t)=D \cos \left[\left(\omega_{0} \pm \Delta \omega\right) t-\varphi+\pi / 2\right] .
$$

It could be seen from (21) that the signal is time reversed compared with the input signal described in (9). On the other hand, the RF leakage from two mixing components can be cancelled at port 4 due to

$f_{\mathrm{RF}}(t)=$

$D^{\prime}\left\{\cos \left[\left(\omega_{0} \pm \Delta \omega\right) t+\varphi-2 \pi\right]+\cos \left[\left(\omega_{0} \pm \Delta \omega\right) t+\varphi-\pi\right]\right\}=0$.

Therefore, this reveals theoretically that the phaseconjugating network can perform time reversal of a chirp signal and a microwave signal with a certain frequency bandwidth. 


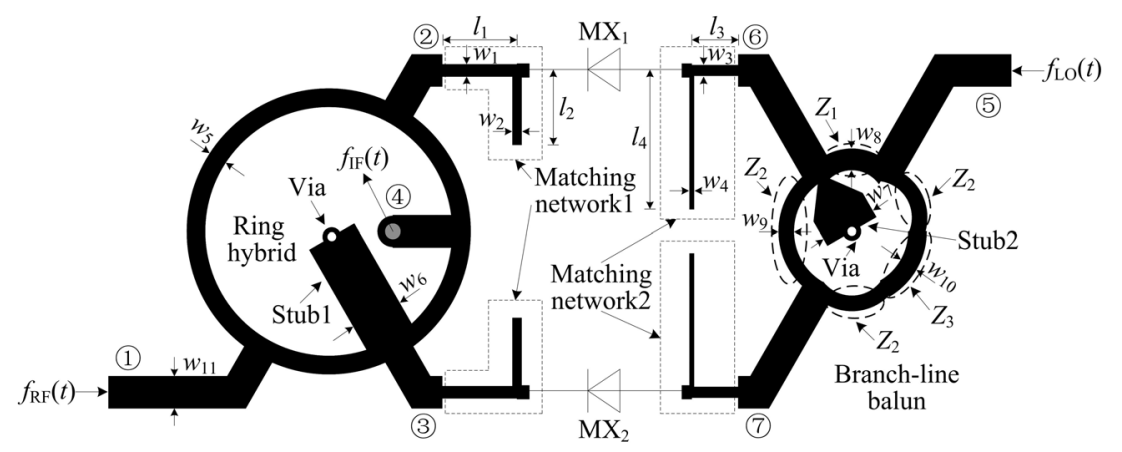

Fig. 2. Schematic diagram of the phase-conjugating network.

\section{Development of the Phase-Conju- gating Network}

As shown in Fig. 2, the mixer diodes are selected as the mixing components. The proposed phase-conjugating network is realized on a microwave substrate with a relative permittivity of 2.65 , a thickness of $0.8 \mathrm{~mm}$, and a loss tangent 0.003 .

Based on the above analyses, the schematic shown in Fig. 2 is numerically analyzed based on Ansoft HFSS and Agilent advanced design system (ADS). The physical parameters of the phase-conjugating network are tabulated in Tab. 1. The ring hybrid and branch-line balun, centered at 5.7 and $11.4 \mathrm{GHz}$, respectively, are first designed. To perform the wideband time reversal, the proposed conjugating network should behave wide operation bandwidth. Therefore, as shown in Figs. 2 and 3, a short-circuited Stub1 is loaded at the port 3 of the ring hybrid to improve the phase bandwidth [16], and Stub2 is attached to the branch-line balun to realize the magnitude compensation [17]. The two stubs are designed as quarter-wavelengths at $5.7 \mathrm{GHz}$ and 11.4 GHz, respectively, and their characteristic impedances are determined based on numerical optimizations. Furthermore, the phase bandwidth of the branch-line balun can be enhanced by using different line impedances of the quarter or half wavelength branch-lines, as illustrated in Fig. 2, where $Z_{1}=63.7 \Omega, Z_{2}=76.6 \Omega$, and $Z_{3}=70.7 \Omega$ after numerical optimizations. For the two proposed structures, it can be seen that for a magnitude imbalance of $\pm 0.5 \mathrm{~dB}$, the magnitude bandwidths are $24.2 \%$ at $5.7 \mathrm{GHz}$ and $25.7 \%$ at

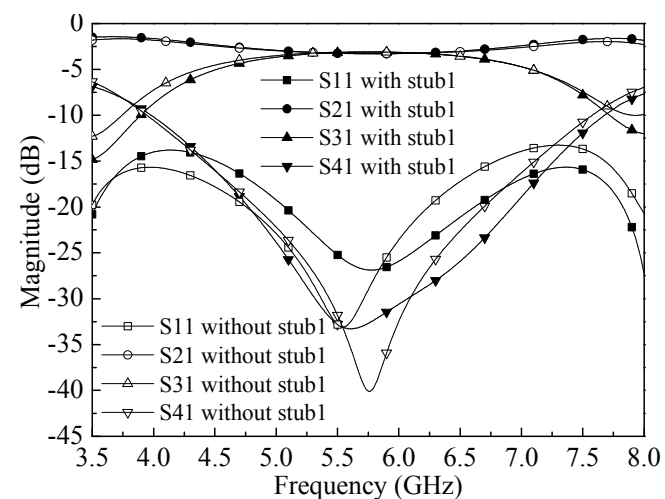

(a)

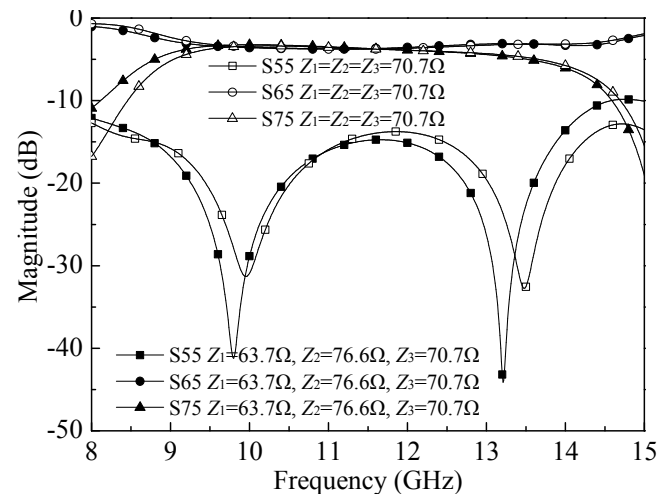

(b)

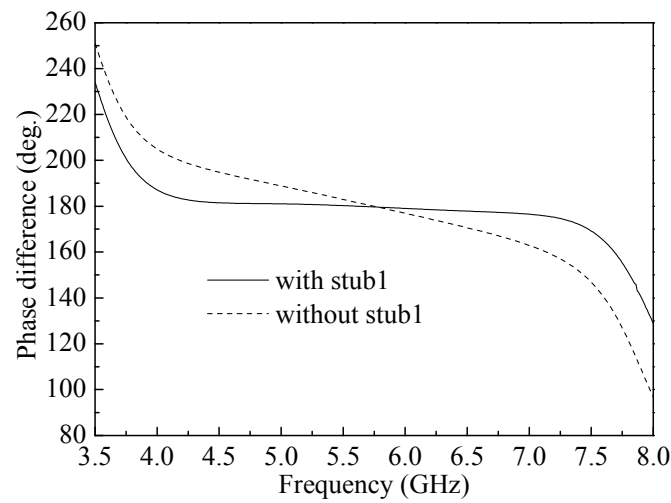

(c)

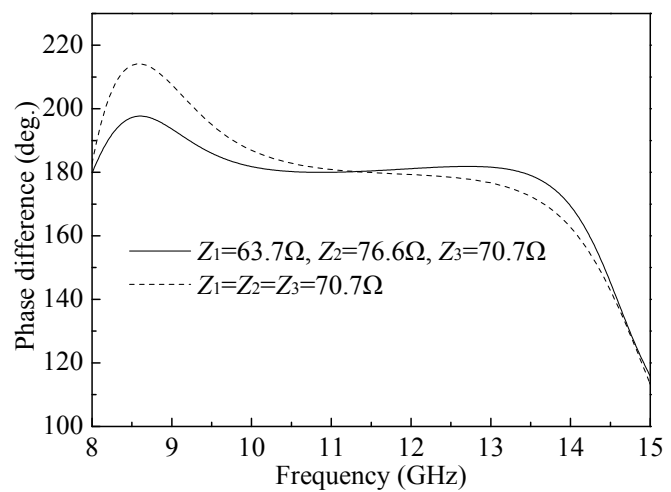

(d)

Fig. 3. Simulated scattering parameters of the ring hybrid and branch-line balun. (a) S-parameter of the ring hybrid. (b) S-parameter of the branch-line balun. (c) Phase difference between port 2 and port 3. (d) Phase difference between port 6 and port 7 . 


\begin{tabular}{|c|c|c|c|}
\hline$l_{1}=4.9$ & $l_{2}=4.85$ & $l_{3}=3.05$ & $l_{4}=9.05$ \\
\hline$w_{1}=0.8$ & $w_{2}=0.6$ & $w_{3}=0.7$ & $w_{4}=0.7$ \\
\hline$w_{5}=1.2$ & $w_{6}=3.4$ & $w_{7}=3.7$ & $w_{8}=1.4$ \\
\hline$w_{9}=1$ & $w_{10}=1.2$ & $w_{11}=2.1$ & \\
\hline
\end{tabular}

Tab. 1. Physical parameters of the phase conjugating network (Unit: $\mathrm{mm}$ ).

$11.4 \mathrm{GHz}$, while referring to $180^{\circ} \pm 3^{\circ}$, the phase bandwidths are $40 \%$ at $5.7 \mathrm{GHz}$ and $33.9 \%$ at $11.4 \mathrm{GHz}$. As shown in Fig. 3, one can see the passive devices exhibit good frequency responses.

As shown in Fig. 2, the operation frequencies of $\mathrm{RF} / \mathrm{IF}$ and LO in this phase conjugating circuit are $5.7 \mathrm{GHz}$ and $11.4 \mathrm{GHz}$, respectively. Here, L shaped matching network are utilized to match the mixer diodes, where the open stubs at the two sides of diodes are designed as quarter-wavelengths at $\mathrm{LO}$ and $\mathrm{RF} / \mathrm{IF}$, respectively, thus presenting circuit loops. As listed in Tab. 1, other parameters of the matching networks are analytically designed and confirmed from numerical simulations. The circuit performance is numerically evaluated based on the monochromatic signal. Notice that though the matching network is designed at the center frequencies, the developed phaseconjugating network can realize good performance within a broad frequency band, as discussed in Sec. 4.

\section{Experimental Validation on the Phase-Conjugating Network}

The studied phase-conjugating network is built on a microwave substrate mentioned above, where two mixer diodes, DMK 2790, are bonded on it. Photograph of the developed network is shown in Fig. 4. The RF signal is generated by Agilent vector network analyzer N9918A, and the LO signal is provided by a harmonic signal source MG3694C. The N9918A is also used to record the output spectrum at port 4 shown in Fig. 2.

The conversion losses versus LO power levels and RF frequencies are first investigated, where the RF power is $-10 \mathrm{dBm}$ and LO frequency is fixed at $11.4 \mathrm{GHz}$. The measured and simulated results are shown in Figs. 5(a) and (b), respectively. From Fig. 5(a), it is seen the minimum conversion loss is $7.1 \mathrm{~dB}$ when the RF frequency is $5.65 \mathrm{GHz}$ with a LO power of $10 \mathrm{dBm}$. Also, when the LO power is $10 \mathrm{dBm}$, Figure $5(\mathrm{~b})$ shows the bandwidth ranges

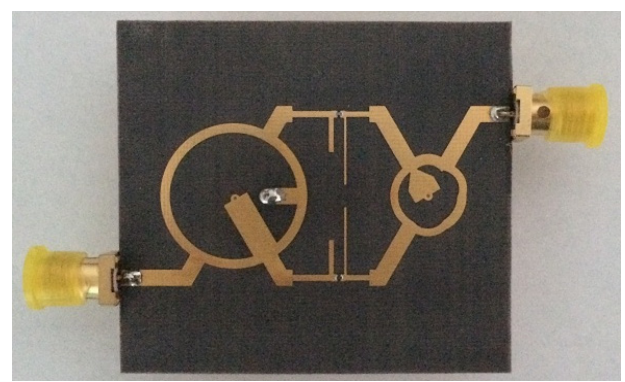

Fig. 4. Photograph of the developed phase-conjugating network.

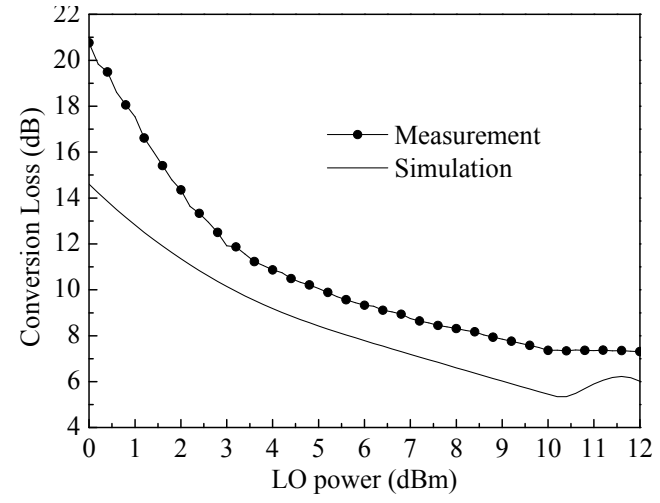

(a)

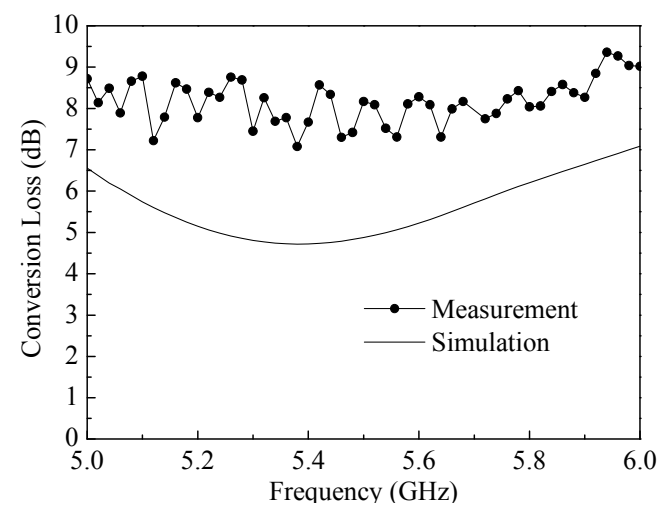

(b)

Fig. 5. Measured and simulated conversion losses. (a) By sweeping the power of local oscillator. (b) By sweeping the RF frequency.

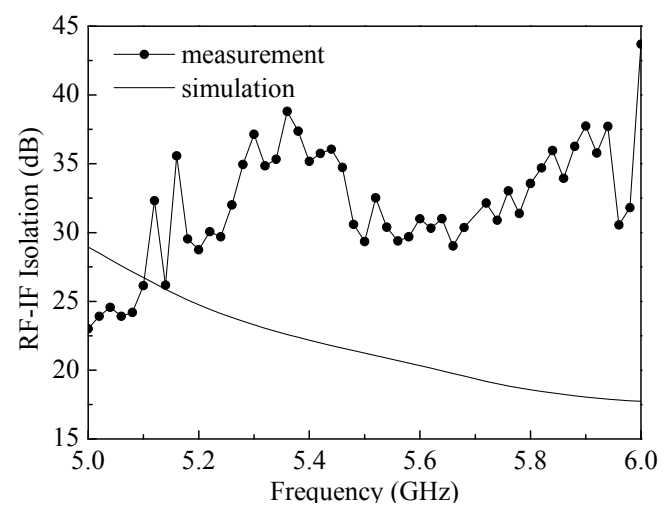

(a)

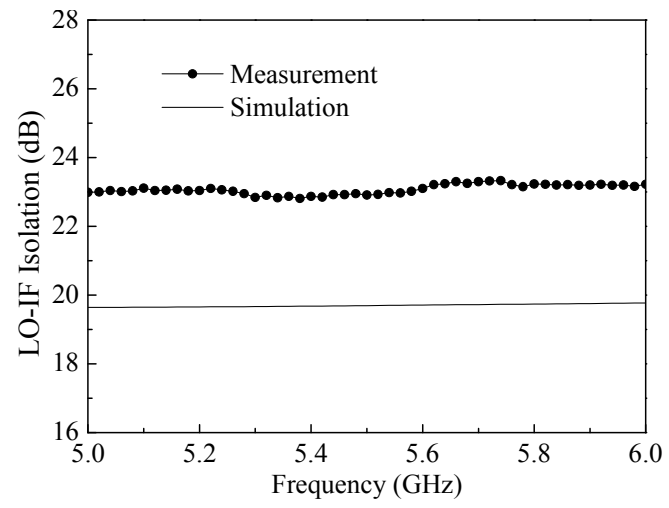

(b)

Fig. 6. Measured and simulated isolation of RF-IF and LO-IF. (a) RF-IF isolation. (b) LO-IF isolation. 


\begin{tabular}{|c|c|c|c|c|c|c|c|}
\hline Refs./Years & Method & Mixer & RF frequency & $\begin{array}{c}\text { RF-IF } \\
\text { separation }\end{array}$ & $\begin{array}{c}\text { Conversion } \\
\text { loss/gain }\end{array}$ & RF-IF isolation & $\begin{array}{c}\text { LO-IF/RF } \\
\text { isolations }\end{array}$ \\
\hline$[10] / 2004$ & $\begin{array}{c}\text { Fundamental } \\
\text { mixing }\end{array}$ & Diode & $5.8 \mathrm{GHz}$ & Yes & $\begin{array}{c}6 \mathrm{~dB}(\text { loss }) \\
(5.7-5.9 \mathrm{GHz})\end{array}$ & $\begin{array}{c}\geq 20 \mathrm{~dB} \\
(5.7-5.9 \mathrm{GHz})\end{array}$ & $\begin{array}{c}\text { Without } \\
\text { mentioned }\end{array}$ \\
\hline$[12] / 2003$ & $\begin{array}{c}\text { Fundamental } \\
\text { mixing }\end{array}$ & FET & $5.8 \mathrm{GHz}$ & Yes & $\begin{array}{c}20 \mathrm{~dB}(\mathrm{gain}) \\
(5.75-5.81 \mathrm{GHz})\end{array}$ & $\begin{array}{c}\geq 15 \mathrm{~dB} \\
(5.75-5.81 \mathrm{GHz})\end{array}$ & $\begin{array}{c}\text { Without } \\
\text { mentioned }\end{array}$ \\
\hline$[13] / 2011$ & $\begin{array}{c}\text { Second harmonic } \\
\text { mixing }\end{array}$ & FET & $5.8 \mathrm{GHz}$ & No & $\begin{array}{c}\leq 5 \mathrm{~dB}(\mathrm{gain}) \\
(5.6-6 \mathrm{GHz})\end{array}$ & $\begin{array}{c}\geq 30 \mathrm{~dB} \\
(5.6-6 \mathrm{GHz})\end{array}$ \\
\hline$[14] / 2015$ & $\begin{array}{c}\text { Fundamental } \\
\text { mixing }\end{array}$ & FET & $3.95 \mathrm{GHz}$ & No & $\begin{array}{c}2 \mathrm{~dB}(\mathrm{loss}) \\
(3.6-4.3 \mathrm{GHz})\end{array}$ & $\begin{array}{c}\text { Without } \\
\text { mentioned }\end{array}$ \\
\hline This work & $\begin{array}{c}\text { Fundamental } \\
\text { mixing }\end{array}$ & Diode & $5.7 \mathrm{GHz}$ & Yes & $\begin{array}{c}8.2 \pm 1.1 \mathrm{~dB}(\mathrm{loss}) \\
(5-6 \mathrm{GHz})\end{array}$ & $\begin{array}{c}\geq 30 \mathrm{~dB} \\
(5-6 \mathrm{GHz})\end{array}$ & $\begin{array}{c}\geq 23 \mathrm{~dB} \\
(5-6 \mathrm{GHz})\end{array}$ \\
\hline
\end{tabular}

Tab. 2. Performance comparisons for the phase conjugating of microwave signal.

from 5 to $6 \mathrm{GHz}$ under an imbalance of $(8.2 \pm 1.1) \mathrm{dB}$, as compared to the simulated results of $(5.9 \pm 1.2) \mathrm{dB}$. The difference between simulations and measurements can be attributed to the simulation model of the diode that is slightly deviated from its actual parameters.

The measured and simulated RF-IF and LO-IF isolations are shown in Fig. 6, where RF and LO signal are the same as presented in Fig. 5(b). It is found the experimental results are better than that from simulations. From Fig. 6(a), one can see the RF-IF isolation from measurements is approximately $30 \mathrm{~dB}$ from 5.2 to $6 \mathrm{GHz}$, as compared to the simulation results of $20 \mathrm{~dB}$. Figure 6(b) shows the LO-IF isolation is better than $23 \mathrm{~dB}$ within this range, where it is approximately $19 \mathrm{~dB}$ from simulations.

For performance comparisons, Table 2 lists some reported results and this demonstrator. Compared with the related contributions, this work uses Schottky diodes to realize fundamental mixing without the DC bias. The RF and IF signal is separated to facilitate observing and analyzing their spectrums. The conversion loss is $8.2 \mathrm{~dB}$ with small imbalance in a relatively broad frequency band. This topology also features good RF-IF and LO-IF isolations within the same frequency range.

For a fixed LO signal, time reversal of a chirp RF signal is studied. The chirp RF signal is generated by the signal source, MG3694C, with a central frequency of $5.7 \mathrm{GHz}$, ranging from 5.68 to $5.72 \mathrm{GHz}$ under a frequency-tuning slope of $K=0.4 \mathrm{MHz} / \mathrm{s}$, and the power level referring to the monochromatic signal is set as $-6.5 \mathrm{dBm}$. In order to observe the time reversal performance, the LO signal provided by the Agilent signal generator E8247C is fixed at $11.35 \mathrm{GHz}$, and the power level is $10 \mathrm{dBm}$. The N9918A is used to observe the spectrum at port 1 and port 4 in Fig. 2. Figure 7 shows the results from measurements and simulations. From Fig. 7(a), the measured RF spectrum agrees with the simulated one in the observed frequency band. Referring to the RF signal, the IF spectrum is ranged from $5.63 \mathrm{GHz}$ to $5.67 \mathrm{GHz}$, which corresponds to the phase conjugating, thus time reversed, as described in Fig. 7(b). In this process, the average conversion loss is approximately $9.5 \mathrm{~dB}$, and the average power of RF signal at port 4 (namely the leakage from ports 1 to 4 , or the isolation between RF and IF) is below $-60 \mathrm{dBm}$. This confirms the theoretical analysis based on (17).

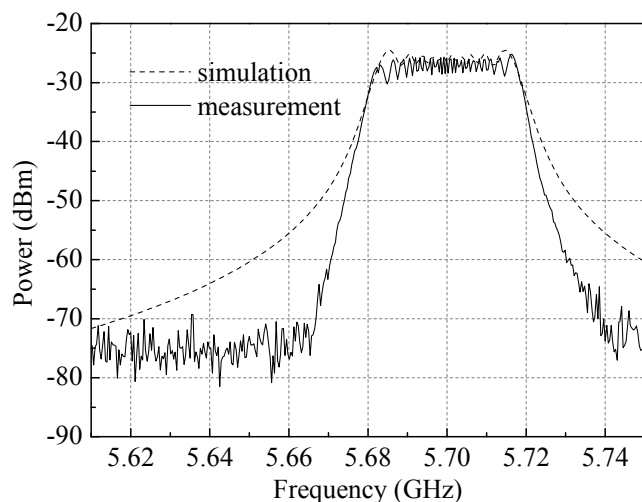

(a)

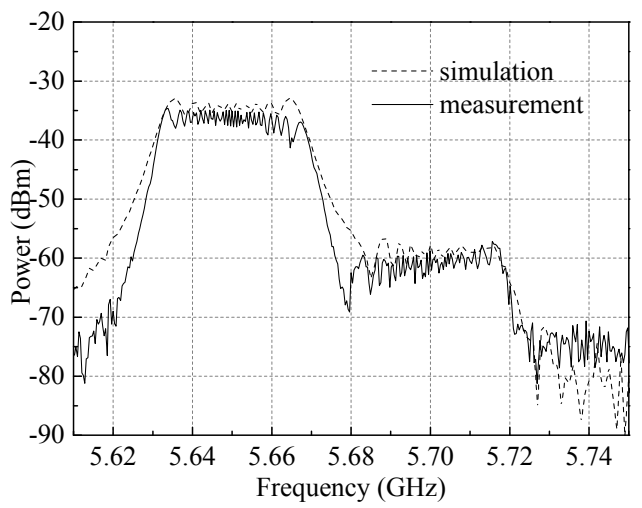

(b)

Fig. 7. The simulated and measured results of chirp signal when the LO frequency is $11.35 \mathrm{GHz}$. (a) Spectrum of the input signal at port 1. (b) Spectrum of the output signal at port 4 .

When the LO frequency is set to $11.4 \mathrm{GHz}$ (i.e. double of the central frequency of the RF signal), the phase conjugating experiment of the chirp signal is further measured. As presented in Fig. 8(a), the RF signal is the same as the one shown in Fig. 7(a). From Fig. 8(b), it is seen the average conversion loss maintains similar levels compared with Fig. 7(b). Figures 7 and 8 illustrate that the chirp signal can be time reversed effectively.

To implement a wideband time reversal of a microwave signal, here we propose a method called dynamically synchronous phase conjugating. Now, assuming the RF signal has a certain frequency band in the spectrum, as described in (9). The LO frequency is double and can dynamically synchronous to the RF frequency. Based on this 


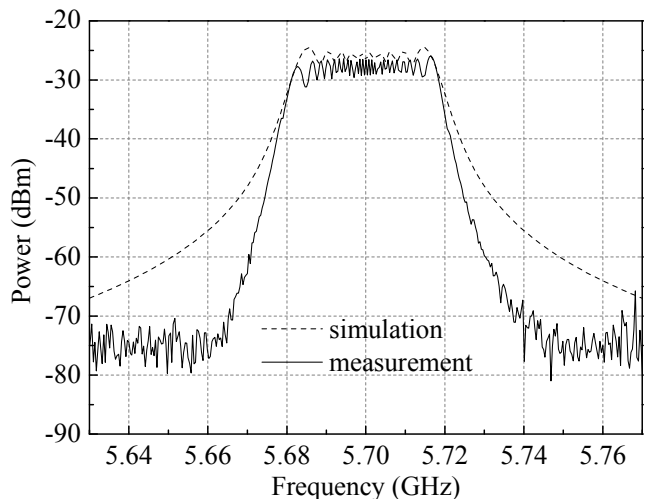

(a)

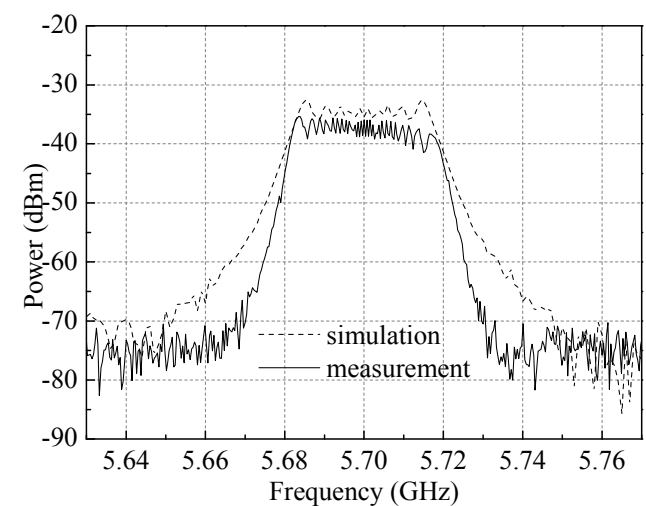

(b)

Fig. 8. The simulated and measured results of chirp signal when the LO frequency is $11.4 \mathrm{GHz}$. (a) Spectrum of the input signal at port 1. (b) Spectrum of the output signal at port 4 .

method, time reversal of a wideband microwave signal can be achieved by using the developed phase-conjugating network. Experimental study is therefore further performed. The RF signal is generated by N9918A, ranging from $4.5 \mathrm{GHz}$ to $7 \mathrm{GHz}$, and its power level is $-10 \mathrm{dBm}$. The LO power is $10 \mathrm{dBm}$, and it is varied synchronously from $9.001 \mathrm{GHz}$ to $14.001 \mathrm{GHz}$, where the extra $1 \mathrm{MHz}$ offset would facilitate the investigation in measurements. For example, when RF frequency is $5.65 \mathrm{GHz}$, the LO frequency is set to $11.301 \mathrm{GHz}$, while when RF frequency is $5.75 \mathrm{GHz}$, the LO frequency is dynamically set to 11.501 GHz. The results are recorded in Fig. 9 for both measurements and simulations. As seen in Fig. 9(a), referring to a $10-\mathrm{dB}$ conversion loss, the bandwidth ranges from $5.4 \mathrm{GHz}$ to $6.25 \mathrm{GHz}$. From Fig. 9(b), the IF-RF isolation is better than $20 \mathrm{~dB}$ from $5.2 \mathrm{GHz}$ to $6.4 \mathrm{GHz}$. Hence, a wideband time reversal of microwave signal is approached. Compared with the simulations, the higher conversion loss from experiments is due to the same reasons as mentioned above.

\section{Conclusion}

A wideband phase conjugating network is studied to time reverse microwave signals. The operation principles and analyses are discussed in the paper. A prototype demonstrator is developed and examined, theoretically and

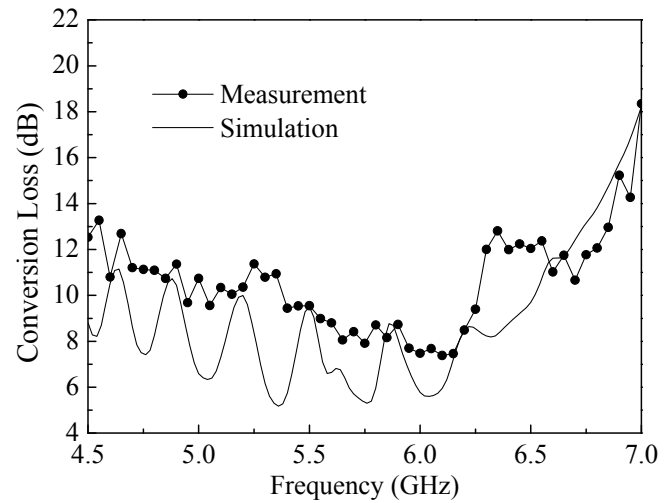

(a)

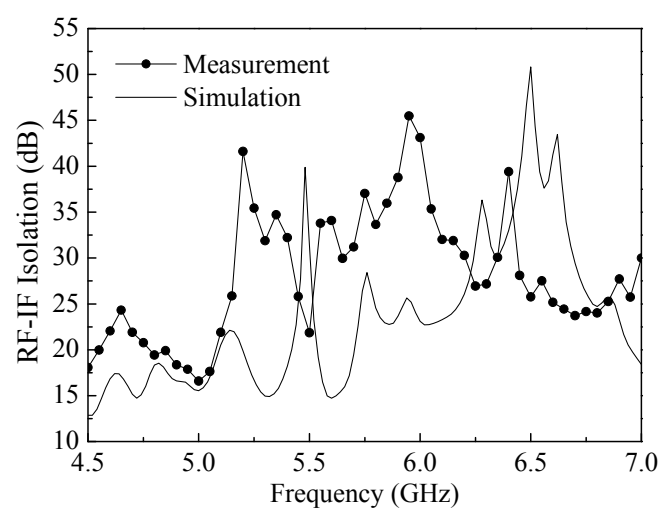

(b)

Fig. 9. The measured and simulated results of synchronous phase conjugating. (a) Conversion loss. (b) RF-IF isolation.

experimentally. Results indicate that a chirp signal with $40 \mathrm{MHz}$ bandwidth, and a microwave sinusoidal signal with a wide frequency range, can be phase conjugated, thus reversed in the time domain. The experiments reasonably match the analysis, thus confirming the presented method.

\section{Acknowledgments}

This work was supported in part by the National Natural Science Foundation of China (NSFC) (61331007, 61271025, and 61601063).

\section{References}

[1] IERO, D. A. M., CROCCO, L., ISERNIA, T. On the role and choice of source polarization in time-reversal focusing of vector fields. IEEE Antennas and Wireless Propagation Letters, 2016, vol. 15, p. 214-217. DOI: 10.1109/LAWP.2015.2438539

[2] LEROSEY, G., ROSNY, J. de, TOURIN, A., et al. Focusing beyond the diffraction limit with far-field time reversal. Science, 2007, vol. 315 , no. 5815 , p. 1120-1122. DOI: $10.1126 /$ science. 1134824

[3] CARMINATI, R., PIERRAT, R., ROSNY, J. de, et al. Theory of time reversal cavity for electromagnetic fields. Optics Letters, 2007, vol. 32, no. 21, p. 3107-3109. DOI: 10.1364/OL.32. 003107 
[4] FINK, M. Time reversal of ultrasonic fields-part I: basic principles IEEE Transactions on Ultrasonics, Ferroelectrics, and Frequency Control, 1992, vol. 39, no. 5, p. 555-566. DOI: $10.1109 / 58.156174$

[5] MUKHERJEE, S., UDPA, L., UDPA, S., et al. Target localization using microwave time-reversal mirror in reflection mode. IEEE Transactions on Antennas and Propagations, 2017, vol. 65, no. 2, p. 820-828. DOI: 10.1109/TAP.2016.2627011

[6] ODEDO, V. C., YAVUZ, M. E., COSTEN, F., et al. Time reversal technique based on spatiotemporal windows for through the wall imaging. IEEE Transactions on Antennas and Propagations, 2017, vol. 65, no. 6, p. 3065-3072. DOI: 10.1109/TAP.2017.269642

[7] LIU, D., KROLIK, J., CARIN, L. Electromagnetic target detection in uncertain media: time-reversal and minimum-variance algorithms. IEEE Transactions on Geoscience and Remote Sensing, 2007, vol. 45, no. 4, p. 934-944. DOI: $10.1109 /$ TGRS.2006.890411

[8] WANG, B., WU, Y., HAN, F., et al. Green wireless communications: a time-reversal paradigm. IEEE Journal on Selected Areas in Communications, 2011, vol. 29, no. 8, p. 1698-1710. DOI: 10.1109/JSAC.2011.110918

[9] CHEN, Y., HAN, F., YANG, Y.-H., et al. Time-reversal wireless paradigm for green internet of things: an overview. IEEE Internet of Things Journal, 2014, vol. 1, no. 1, p. 81-98. DOI: 10.1109/JIOT.2014.2308838

[10] LEONG, K. M. K. H., WANG, Y., ITOH, T. A full duplex capable retrodirective array system for high-speed beam tracking and pointing applications. IEEE Transactions on Microwave Theory and Techniques, 2004, vol. 52, no. 5, p. 1479-1489. DOI: 10.1109/TMTT.2004.827025

[11] GOSHI, D. S., LEONG, K. M. K. H., ITOH, T. A sparse retrodirective transponder array with a time shared phaseconjugator. IEEE Transactions on Antennas and Propagation, 2007, vol. 55, no. 8, p. 2367-2372. DOI: 10.1109/TAP.2007.901852

[12] MIYAMOTO, R. Y., LEONG, K. M. K. H., JEON, S.-S., et al. Digital wireless sensor server using an adaptive smart-antenna/ retrodirective array. IEEE Transactions on Vehicular Technology, 2003, vol. 52, no. 5, p. 1181-1188. DOI: 10.1109/TVT.2003.816610

[13] CHIU, L., XUE, Q., CHAN, C. H. A 4-element balanced retrodirective array for direct conversion transmitter. IEEE Transactions on Antennas and Propagation, 2011, vol. 59, no. 4, p. 1185-1190. DOI: 10.1109/TAP.2011.2109355

[14] TSAI, J.-W., SHIAU, C.-Y., MA, T.-G. Dual-band retrodirective array with integrated reflection-type and phase conjugating array using synthesized transmission lines. In Proceedings of the 4th Asia-Pacific Conference on Antennas and Propagation (APCAP). Bali Island (Indonesia), 2015, p. 185-186. DOI: 10.1109/APCAP.2015.7374326

[15] RICHARDS, M. A. Fundamentals of Radar Signal Processing. New York (USA): McGraw-Hill, 2005 (chapter 1) ISBN: 0071444742

[16] PIERNAS, D., HAYASHI, H., NISHIKAWA, K., et al. Improvement of the design of $180^{\circ}$ rat-race hybrid. Electronics Letters, 2000, vol. 36, no. 12, p. 1035-1036. DOI: 10.1049/el: 20000753

[17] LI, J.-L., SHAO, W., WANG, J.-P., et al. Microwave slow-wave structure and phase-compensation technique for microwave power divider. Radio Engineering, 2014, vol. 23, no. 1, p. 214-221. ISSN $1210-2512$

\section{About the Authors ...}

Cheng-Guang SUN received the B. S. degree in Electronic Engineering and M. S. degree in Physical Electronics from the Hebei University of Technology, Tianjin, China, in 2003 and 2006, respectively. Since April 2006, he was a faculty member with the Institute of Antenna and Microwave Techniques, Tianjin University of Technology and Education, Tianjin, China. Currently, he is working toward the Ph.D. degree at the School of Physics, University of Electronic Science and Technology of China (UESTC). His research interests include microwave circuits and systems, time reversal electromagnetics, etc.

Jia-Lin LI received the M. S. degree from the University of Electronic Science and Technology of China (UESTC), Chengdu, China, in 2004, and the Ph. D. degree from the City University of Hong Kong, Hong Kong, in 2009, both in Electronic Engineering. From September 2005 to August 2006, he was a Research Associate with the Wireless Communication Research Center, City University of Hong Kong, Hong Kong. Since September 2009, he has been with the School of Physics, UESTC, where he is currently a Professor. His research interests include microwave/ millimeter-wave antennas and arrays, circuits and systems, and so on. 